\title{
„Peak Capitalism“? Wachstumsgrenzen als Grenzen des Kapitalismus
}

\author{
Die nachfolgenden Überlegungen erläutern eine These, die bei vielen Zeitgenossinnen und \\ Zeitgenossen auf Unverständnis, Widerspruch oder sogar entschiedene Abwehr stoßen \\ wird. Verkürzt lautet sie: Der Kapitalismus befindet sich auf dem absteigenden Ast. Denn er \\ steckt nicht nur in einer tief greifenden, strukturellen Krise seiner Akkumulationsdynamik, \\ welche sich in einem säkularen Niedergang von Investitionen im Verhältnis zu den erzielten \\ Gewinnen und daher in einer Verlangsamung des globalen Wirtschaftswachstums ausdrückt. \\ Dies ist die Krise des Kapitalismus als sozioökonomisches System. In einer existenziellen \\ Krise befindet sich der Kapitalismus im dritten Jahrhundert seiner Existenz aber vor allem \\ als ein ökologisches Weltsystem - und für diese Krise gibt es, anders als für die zyklischen \\ Akkumulationskrisen, keine systemimmanente Lösung.
}

BIRGIT MAHNKOPF

\section{Einleitung}

Die These von einem „Peak Capitalism“ (Mahnkopf 2013) - dass die kapitalistische Entwicklung also ihren Zenit erreicht habe - mag an den schon häufiger vorhergesagten „Zusammenbruch des Kapitalismus“ erinnern. Darauf wird in der Regel mit dem Verweis reagiert, der Kapitalismus habe im Verlauf des 20. Jahrhunderts doch unter Beweis gestellt, dass er zur Selbsterneuerung fähig sei - warum also sollte dies im 21. Jahrhundert anders sein? Ein „grüner Kapitalismus" könnte doch "pfleglicher" mit der sogenannten „Umwelt" verfahren, ohne damit Eigentums- oder Herrschaftsverhältnisse infrage zu stellen. Dagegen wird im Folgenden argumentiert, dass der Kapitalismus als ein sozio-ökologisches Weltsystem (vgl. Moore 2012), das alle Natur seinem Bewegungsgesetz unterwirft, heute an absolute Grenzen stößt. An den „planetarischen Grenzen“ von biophysischen Systemen erweist sich die spezifische Eigenart des Kapitalismus, nämlich seine Missachtung der biophysischen wie der sozialen Schranken - die in früheren Phasen seiner Entwicklung die revolutionäre Kraft „schöpferischer Zerstörung “ begründet hat -, als ein selbstdestruktiver Mechanismus und als praktisch unüberwindbares Hindernis für eine neue Phase der Akkumulation.

Ob nach dem Erreichen des „Höhepunktes“ des Kapitalismus als ein weltökologisches System die abfallende Kurve flach gehalten werden kann oder es in einer steilen Kurve bergab geht - ob also eine Evolution (oder Transformation) des Systems noch möglich ist -, das können wir heute nicht wissen. Mit großer Sicherheit aber bricht ein sozioökonomisches System nicht einfach zusammen. Mit der existenziellen Krise des Kapitalismus als eines weltökologischen Systems werden jedoch Kippschalter („tipping points“, vgl. Rockström et al. 2009) aktiviert, die gravierende Veränderungen unvermeidlich machen. Diese werden ein Resultat der kumulativen Wirkungen sein, die sich aus dem Widerstand arbeitender (aber nicht unbedingt lohnabhängiger) Klassen, der unwiderruflichen Veränderung von ökologischen Systemen und dem Wirken von Marktprozessen ergeben.

\section{Das „Wachstumswunder“ im Kapitalozän}

Mit dem Kapitalismus ist seit dem „langen 16. Jahrhundert“ (in der Zeit von 1492 bis 1648) ein soziales und ökologisches System entstanden, das die menschliche wie die außermenschliche Natur einer Zweck-Mittel-Rationalität unterwirft. Alles wird zum Material ökonomischer Verwertung gemacht; das schließt auch den Körper und die Psyche der Menschen mit ein. Doch nicht nur die biologischen Grundlagen des Lebens, sondern auch die geologischen und 
klimatischen Grundbedingungen des Planeten Erde werden heute nachhaltig von den Aktivitäten der Menschen beeinflusst. Daher sprechen die Erdwissenschaftler von einer neuen geologischen Epoche, vom „Anthropozän“ (Crutzen 2002). Ein „Menschenzeitalter“ hat die mehr als 12.000 Jahre währende Epoche des „Holozäns“ abgelöst, also diejenige Phase in der Erdgeschichte, die durch eine bemerkenswerte langfristige Stabilität bei der Temperaturentwicklung und hinsichtlich der Ablagerung von Emissionen in der Atmosphäre gekennzeichnet war. Es hatte sich ein kleines „Temperaturfenster“ geöffnet, in dem menschliche Entwicklung auf dem Planeten möglich wurde. Doch innerhalb der letzten drei Jahrhunderte sind sowohl der Meeresspiegel als auch die globale Durchschnittstemperatur sowie die Zahl der Menschen auf dem Planeten in zuvor unvorstellbarem Umfang gestiegen; zugleich ist die Festlandsoberfläche geschrumpft und unzählige Arten sind ausgestorben. Dieser erdgeschichtliche Wandel ist wesentlich auf Wirkungen des mittlerweile weltumspannenden ökonomischen Systems des Kapitalismus zurückzuführen. Daher sollte die neue Erdepoche des von Geologen so bezeichneten Anthropozäns korrekter als „Kapitalozän“ (Altvater 2014) bezeichnet werden.

\subsection{Wachsender Energie- und Rohstoffverbrauch}

Im Verlauf dieser letzten drei Jahrhunderte ist es tatsächlich gelungen, die Erde - wie es die jüdisch-christliche Botschaft gebietet - „dem Menschen untertan zu machen“, sie nach menschlichen Vorstellungen und Wünschen zu formen und umzugestalten. Im Verlauf der von Europa ausgehenden kapitalistischen Welteroberung wurden die ökonomischen Wachstumsraten seit dem Ende des 18. Jahrhunderts beständig gehoben, real und pro Kopf im Durchschnitt um etwa 2,2\% pro Jahr (Maddison 2001; vgl. auch Pikkety 2014). Das hat in den vergangenen zweieinhalb Jahrhunderten zu einer Verdoppelung des monetären Pro-KopfEinkommens von einer Generation zur nächsten geführt. Ermöglicht wurde dieses „Wachstums-Wunder“ durch den Rückgriff auf fossile Energieträger und deren hohen EROI (energy return on energy invested): die hohe „Energierendite“ auf die eingesetzte Energie. Denn nur mit Hilfe billiger Energie (zunächst Kohle, dann Erdöl und Erdgas) gelang es, an die Rohstoffe zu kommen, die Voraussetzung sind für Industrialisierung und Urbanisierung, an Metalle und nichtenergetische Mineralien (Bardi 2013), darunter so scheinbar unerschöpfliche wie der Sand, der heute wie viele andere Stoffe seinen „Peak“, also das Höchstproduktionsplateau, bereits überschritten hat. Billige fossile Energieträger waren nicht zuletzt eine wesentliche Voraussetzung für die Produktivitätssteigerungen, die sich in der Zeit nach dem Zweiten Weltkrieg unter dem Label der „Grünen Revolution“ in der Landwirtschaft abgespielt haben. Nur durch den Einsatz von energieintensiven Maschinen, von ölbasierten Pflanzenschutz- und Düngemitteln wie dem wasserlöslichen Phosphat sowie durch den verschwenderischen Umgang mit dem ebenfalls knapp werdenden Wasser konnten die Nahrungsmittelpreise seit den 1970er Jahren bis zu Beginn der 2000er Jahre auf ein historisches Tiefstniveau fallen.

Wirtschaftswachstum ist nur der volkswirtschaftlich sichtbare Ausdruck eines Systems, in dem Geld als Kapital fungiert und durch einen permanenten Investitionskreislauf vermehrt, also akkumuliert oder verwertet werden soll. Dies geschieht mit Hilfe der sozialen und ökonomischen Treiber Profitprinzip, Zinseszins und Renditezwang. Weil Geld als Kredit immer einen Überschuss in Form von Zinsen und Renditen erwirtschaften muss, kann die Maßlosigkeit, eine ins Unendliche strebende Bewegung, als die Wesenslogik des Kapitalismus verstanden werden. Unter den Bedingungen verallgemeinerter kapitalistischer Konkurrenz müssen allerdings auch die Aufwendungen für diese Verwertungsprozesse (Rohstoffe und Energie zumal) permanent erhöht werden - und das treibt die Kosten des vorgeschossenen Kapitals in die Höhe. Vor allem aber müssen sich immer neue Anlagemöglichkeiten finden lassen, in denen die von den Kapitaleignern gewünschte Rendite realisiert werden kann. Gelingt dies nicht, reichen auch eine Verbilligung des Maschineneinsatzes, technischer Fortschritt und eine Anhebung der Gewinne, die sich aus der Ausbeutung der Arbeitskraft ziehen lassen, nicht aus, um die Wachstumsdynamik auf einem fortwährend hohen Niveau zu halten.

\subsection{Zunahme sozialer Verwerfungen}

Werden die Wachstumsraten gehoben, so wie dies insbesondere während der letzten Jahrzehnte in nahezu allen Regionen des Globus der Fall war, bedingt dies einen höheren Energie- und Materialeinsatz, doch dank arbeitssparender technologischer Innovationen nicht unbedingt einen Mehrbedarf an Arbeitskräften. Für entwickelte Industrieländer ist bekannt, dass spürbare Wirkungen am Arbeitsmarkt eigentlich erst ab einer jährlichen Wachstumsrate des Bruttoinlandsprodukts von mehr als 2,3 \% zu erwarten sind - es sei denn, Arbeitnehmerinnen und Arbeitnehmer können, wie dies heute nicht nur in den Ländern des globalen Südens, sondern teilweise auch in Deutschland oder in den USA der Fall ist, durch den Abbau sozialer Schutzmechanismen dazu gezwungen werden, selbst zu Hungerlöhnen $\mathrm{zu}$ schuften. Blind gegenüber allen gesellschaftlichen und ökologischen Folgen seiner beständig wachsenden Verwertungstätigkeit geht es in dem sinnlosen Prozess der Selbstverwertung des Kapitals ja schließlich darum, durch möglichst rationell organisierte Warenproduktion möglichst hohe Profite zu realisieren - und dieses Ziel gebietet allemal, den Anteil der Lohnarbeit am Produktionsprozess schrittweise zu verringern.

So verwundert es nicht, dass mit dem wirtschaftlichen Wachstum zugleich die globale Arbeitslosigkeit, die informelle Ökonomie und die soziale Ungleichheit angewachsen sind. Besonders rasant verlief diese Entwicklung seit den 1990er Jahren, als in der zweiten Phase der neoliberalen Globalisierung eine Öffnung der Volkswirtschaften zum 
Weltmarkt mit besonderer Radikalität betrieben wurde. Zwischen 1990 und 2010 ist die Einkommensungleichheit der Haushalte sowohl in den Ländern mit niedrigem, mit mittlerem wie mit hohem Volkseinkommen deutlich gestiegen (vgl. UNDP 2014, S. 80). In der Regel wird auf der Plusseite gesteigerten ökonomischen Wachstums verbucht, dass viele Menschen heute besser ernährt sind und eine bessere Gesundheitsversorgung erhalten, daher auch länger leben als früher, dass der Bildungszugang sich deutlich verbessert hat und soziale Sicherungssysteme auch armen und sehr armen Bevölkerungsschichten zugänglich sind. Doch liegt der Zusammenhang zwischen ökonomischem Wachstum und diesen zweifellos zentralen Indikatoren gesellschaftlichen Fortschritts keineswegs auf der Hand. Wäre dies der Fall, müssten die Fortschritte bei Bildung, Gesundheit und Ernährung in den Ländern mit besonders hohen Wachstumsraten (und mit einem höheren Einkommensniveau) auch entsprechend stark ausgefallen sein; doch davon kann nicht die Rede sein (vgl. UNDP 2014, S. 128ff.). Ökonomisches Wachstum ist keineswegs der entscheidende Treiber für Verbesserungen bei Bildung, Gesundheit und Ernährungssituation; wichtiger sind die Verteilung der Einkommen, Qualität und Quantität öffentlicher Ausgaben, doch auch Mitsprachemöglichkeiten und Korruptionskontrolle bei der Bereitstellung von öffentlichen Diensten (vgl. Acemoglu/Robinson 2012; Dreze/Sen 2013).

Dennoch kreist die öffentliche Debatte heute nicht um die Frage, wie sich die Verteilung von Ressourcen und von Lebenschancen auf dem Planeten Erde gerechter organisieren ließe. Selbst diejenigen Ökonomen, die öffentlich ihre Befürchtung äußern, dass wir uns in einer „großen Rezession“ (Paul Krugman) oder sogar inmitten einer „säkularen Stagnation“ (Laurence Summers) befinden, bewegt vor allem die Frage, wie die Wachstumskräfte des Kapitalismus wiederbelebt werden könnten. Hinsichtlich der Instrumente zur Ankurbelung des stotternden Wachstumsmotors gibt es zwischen neoklassisch orientierten, neoliberalen und keynesianisch argumentierenden Ökonomen erhebliche Unterschiede; doch teilen alle diese Ökonomen letztlich die Grundüberzeugung, dass das globale kapitalistische Wirtschaftssystem zwar vorübergehend quantitativ schrumpfen könne (ebenso wie es sich zuvor ausgedehnt hat), dass es aber nach überwundener Krise in seiner Qualität unverändert bleibe. Stets geschieht dies unter der Annahme eines zyklischen Musters ökonomischer Entwicklung, welches von Prosperität über eine Krise zurück zur Prosperität führt.

Fragen, die die Zukunftsfähigkeit des Kapitalismus als eines sozial-ökologischen Weltsystems betreffen, werden indes schlichtweg ausgeblendet: Was geschieht mit dem Produktions- und Konsumsystem des Kapitalismus, wenn elementare Voraussetzungen desselben zusehends unterhöhlt werden - insbesondere wenn die Aneignung und nachfolgende Plünderung von leicht zugänglichen und reichen Vorräten an mineralischen und agrarischen Rohstoffen sowie die Verfügbarkeit von sauberem Wasser schwierig, ja für wachsende Teile der Weltbevölkerung nahezu unmög- lich wird? Wie ließen sich die kapitalistische Produktionsund Lebensweise und die darauf aufbauenden politischen Systeme stabilisieren, wenn das Wachstum zum Erliegen käme, etwa deswegen, weil nur billige Energieträger (mit einem hohen EROI) hohe Renditen, hohe Investitionen und daher hohe Wachstumsraten ermöglichen?

\section{Konturen der säkularen "großen Krise“ des Kapitalismus}

Vieles spricht dafür, dass wir es gegenwärtig weder mit einer normalen, zyklischen, noch mit einer auf wenige Länder begrenzten Krise zu tun haben. Wir erleben zum einen eine tiefe realökonomische Krise, die Ungleichgewichte im Außenhandel zum Ausdruck bringt, welche sich unter den Bedingungen globalen Wettbewerbs bestenfalls von einer Region in andere verschieben, nicht aber grundsätzlich einebnen lassen. Diese Krise ist zum anderen eine Krise des Geldes und der Finanzen und zugleich eine epochale Krise der Arbeit. Aus der von David Ricardo so bezeichneten „Überschussbevölkerung “, die unter den Bedingungen eines großen Sektors von Subsistenzlandwirtschaft - der "freigesetzte“ Arbeitskräfte immer wieder aufgenommen und versorgt hat - eine wichtige ökonomische Funktion als „Reservearmee des Kapitals“ in Prozessen der Industrialisierung übernahm, ist längst eine „überflüssige Menschheit“ (Davis 2007) geworden. Wer zu diesem Teil der Menschheit gehört, muss in der Regel zwar hart arbeiten, um seinen Lebensunterhalt zu sichern, doch geschieht dies in wachsendem Maße in der informellen Ökonomie, d.h. unter Bedingungen, in denen „ordentliche“, also formell geregelte (und erst recht sozial geschützte) Arbeitsverhältnisse die Ausnahme und nicht die Regel sind. Anders als in seiner historischen Vergangenheit erwies sich der Kapitalismus nach seinem Sieg über das konkurrierende System des „realen Sozialismus“ als vollkommen unfähig, den Zustrom von vielen Millionen neuen Lohnarbeitern aus der ehemals vom Weltmarkt abgeschirmten „Zweiten Welt“ zu absorbieren; als unfähig erwies er sich auch, die durch seine eigene Dynamik produzierten „Überflüssigen“ aus der „Dritten“ (und später auch aus der „Ersten“) Welt in wertschaffende Produktionsprozesse zu integrieren.

Vor allem aber ist die gegenwärtige Krise eine ökologische. $\mathrm{Zu}$ deren Facetten zählen nicht nur die für viele Menschen bereits drastisch erfahrbaren Folgen des Klimawandels und die mit ihnen einhergehende Krise in der Versorgung mit sauberem Wasser. Dazu gehört auch die - mangels Wissens und/oder Interesse - weit weniger beachtete Krise der Biodiversität. Nicht zuletzt ist die globale Krise des Kapitalismus auch eine Energie- und Rohstoffkrise. Deren spezifische Dynamik resultiert aus drei eng miteinander verknüpften Treibern: erstens den Verschiebungen in den internationalen Machtkonstellationen, welche dazu führen, dass die Ressourcenansprüche der frühindustrialisierten Kernländer des 
Kapitalismus heute auf einen wachsenden „Ressourcennationalismus" rohstoffreicher Länder (in der Regel aus dem globalen Süden) und auf die Begehrlichkeiten aufstrebender neuer Industrieländer stoßen; zweitens dem vermutlich noch bis zur Jahrtausendmitte anhaltenden Bevölkerungswachstum in den Ländern des Südens und den sehr hohen Ressourcenansprüchen der (schrumpfenden) Bevölkerungen in den alten Industrieländern; sowie drittens den zunehmenden Migrationsbewegungen, die eine Folge sowohl der epochalen Krise der Arbeit wie der Klima- und Wasserkrise sind, doch gleichermaßen durch die zunehmend gewalttätig ausgetragenen Konflikte um den Zugang zu Rohstoffen angetrieben werden.

Hinzu kommt die absehbare Verschärfung einer heute in vielen Weltregionen bereits offensichtlichen Krise von Nahrungsmittelproduktion und -verteilung. Ausgelöst wird diese durch das Anwachsen und die Verstädterung der Weltbevölkerung, die sinkenden Ertragsraten vieler Anbauflächen für Grundnahrungsmittel und das „land grabbing“ finanzstarker Investoren, das die bäuerliche Wirtschaft in vielen Teilen der Welt zur Kapitulation treibt. Keineswegs hilfreich ist es in diesen Zeiten der säkularen Krise, dass vielerorts, nicht allein in der Europäischen Union, das Vertrauen in die Demokratie schwindet und der „Mafia-Kapitalismus“ zu einem weitverbreiteten Phänomen geworden ist.

Die ökonomischen, ökologischen, sozialen und politischen Dimensionen dieser Jahrhundertkrise sind also aufs engste miteinander verknüpft. Im Einzelnen fällt es daher schwer, auszumachen, was etwa soziale oder ökologische Ursachen und was ökonomische, soziale oder ökologische Folgen sind. Die Ausgangslage für eine zeitgemäße und der Komplexität des historischen Geschehens angemessene Krisenanalyse ist daher nicht sonderlich günstig. Zwar signalisieren Gleichzeitigkeit und Interdependenz der angesprochenen Krisendimension eine epochale Krise des Kapitalismus als globales sozioökologisches System, welche leicht in eine Krise der menschlichen Zivilisation einmünden könnte. Dennoch sieht die übergroße Mehrzahl der Ökonomen nach wie vor keinen Anlass, sich mit den Auswirkungen wirtschaftlichen Handelns auf die menschliche und außermenschliche Natur zu beschäftigen. Bis heute weigert sich die Disziplin zur Kenntnis zu nehmen, dass alle ökonomischen Transaktionen zugleich unumkehrbare Transformationsprozesse von Energieund Stoffströmen darstellen. Doch auch viele Ansätze kritischer Gesellschaftstheorie und emanzipatorischer politischer Praxis beschäftigen sich, wenn überhaupt, oft nur am Rande mit den ökologischen Voraussetzungen ökonomischer Transaktionen und sozialer Interaktionen und ihren Folgen für biophysische Systeme. Das mag damit zusammenhängen, dass dem hegemonialen Paradigma des Wachstums, dem die Ökonomie als Disziplin verpflichtet ist, in der Gesellschaftstheorie ein ebenso wirkungsmächtiges Paradigma entspricht - nämlich das der gesellschaftlichen Stabilität. Dieses aber ist seinerseits, zumal in modernen, demokratisch verfassten Gesellschaften, eng an den Fetisch ökonomischen Wachstums gekoppelt. Das ideologische Konstrukt Wachstum ist längst $\mathrm{zu}$ einer gesellschaftlichen Norm geworden. Es hat sich in unzählige materielle und immaterielle Institutionen eingeschrieben, die selbst gesetzten Regeln folgen: Es werden Gesetze erlassen, Programme formuliert und bindende Vereinbarungen geschlossen, die wie durch einen Zauber vor der Einsicht schützen sollen, dass die nach den Verwüstungen des Zweiten Weltkriegs auf der Grundlage spottbilligen Öls realisierten hohen Wachstumsraten endgültig vorbei sind.

\section{Die Beudeutung natürlichen Reichtums für die kapitalistische Akkumulationsdynamik}

Unsere meist auf den globalen Norden konzentrierte Perspektive lässt vergessen, dass es sowohl in der Geschichte des „modernen Westens“ wie in einer Vielzahl nicht-westlicher Kulturen Vorstellungen von Arbeit, Innovation, Wissen und von einem gelingenden, „guten Leben“ gegeben hat und auch heute noch gibt, welche sich grundlegend von dem „wirtschaftlich gelebten Leben“ des modernen Kapitalismus unterscheiden. Nur im Kapitalismus wird die biophysikalische Welt als eine Menge von verfügbaren Rohstoffen für Zwecke der profitablen Ausbeutung behandelt, nur hier kann alles zu einer Ressource der Kapitalakkumulation werden: fruchtbarer Boden, mineralische Stoffe, das „Lebensgut“ Wasser, die menschliche Arbeitskraft und schließlich selbst die Subjektivität des Individuums. Die Betrachtungsweise der Welt als einer Ansammlung ausbeutbarer Rohstoffe bedingt aber notwendigerweise, dass auch Menschen als ein Rohstoff behandelt werden. Mit diesen wird „pfleglich“ umgegangen, solange sie sich für den kapitalistischen Verwertungsprozess als nützlich erweisen. Ist dies jedoch nicht (mehr) der Fall, können auch Menschen zu „Müll“ werden, zu überflüssigem Ballast, der Sozialsysteme „belastet“; oder sie werden dort, wo deren Netze viel zu löchrig sind, als dass sie Halt vor dem Absturz gewährleisten könnten, in die „Senken“ der Slums dieses Planeten „entsorgt“ (vgl. Davis 2007).

In der Geschichte kapitalistischer Weltbeherrschung ist es allerdings bislang noch stets gelungen, die „Unordnung “ und das Chaos, welche mit der Degradation und Zerstörung der menschlichen wie der außermenschlichen Natur einhergehen, zu externalisieren, d.h. auf schwächere Marktteilnehmer, auf entlegene Territorien, in die Ozeane oder in die Atmosphäre zu verlagern. Doch können wir uns das Wirken der wie eine „Weltvernichtungsmaschine“ (Konicz 2012) funktionierenden kapitalistischen Akkumulationsdynamik tatsächlich als einen unendlichen Prozess vorstellen? Oder muss nicht auf dem begrenzten Terrain des Planeten mit absoluten Grenzen gerechnet werden, die sich aus den Gesetzen der Thermodynamik und aus jenen der Evolution ergeben?

Tatsächlich basierte die Akkumulationsdynamik des historischen Kapitalismus seit seiner Entstehung auf der Aneignung und nachfolgenden Plünderung von „Gratisleistungen 
der Natur". Mit diesem Begriff hat Karl Marx elementare Voraussetzungen des Kapitalismus bezeichnet, die dieser nicht selbst hervorbringen musste: kostengünstige und ertragreiche Energieträger, nicht-energetische Mineralien, agrarische Rohstoffe und billige Arbeitskräfte, die noch nicht in den kapitalistischen Verwertungszusammenhang einbezogen waren (vgl. Marx 1970, S. 630). Das Vorhandensein und die kostengünstige Aneignung „natürlichen Reichtums“ ist also ebenso eine Grundvoraussetzung der kapitalistischen Akkumulationsdynamik - und des von Adam Smith gepriesenen, aus der Arbeitsteilung erwachsenden „Wohlstands der Nationen“ wie die Verfügbarkeit von „Abfallräumen“ für die von Menschen nicht mehr verwendbaren Stoffe.

Die Expansion von Warenproduktion und Warenaustausch zunächst in Europa und in der sogenannten „Neuen Welt" der beiden Amerikas, später auch in anderen Weltregionen, war freilich immer begleitet von ökologischen Krisen, und oft gingen solche Krisen Hand in Hand mit der Zerstörung vorkapitalistischer Sozialsysteme. Allerdings waren die so erzeugten Umweltschäden lange Zeit entweder eher geringfügig, lokal begrenzt oder temporärer Natur. In der Regel haben lokale ökologische Krisen sogar wesentlich dazu beigetragen, dass die kapitalistische Dynamik eine neue Entwicklungsrichtung nehmen konnte. So führte in der zweiten Hälfte des 17. Jahrhunderts eine Verknappung von Holzkohle - einer Schlüsselenergie für die frühe Metallverarbeitung und die Glas- und Zuckerindustrie - zur Nutzung der „nicht-konventionellen“ Energiequelle Steinkohle, zunächst in England, dann in anderen europäischen Ländern und schließlich weltweit (vgl. Moore 2010a). Der Energieertrag dieses fossilen Energieträgers aber war wesentlich höher als derjenige von "konventioneller“ Holzkohle; zusammen mit der Entwicklung von geeigneten Technologien zur Transformation dieses Brennstoffs in nützliche Arbeit konnte die neuartige Nutzung des Brennstoffs Kohle dann zur entscheidenden Voraussetzung für die anschließende industrielle Revolution werden. Dieser Akt menschlicher Emanzipation von den „Schranken der Natur" (Sombart 1987, S. 1126ff.) wurde freilich begleitet von harten sozialen Konflikten zwischen Landbevölkerung und Obrigkeit. Denn im Interesse wichtiger Gewerbe der merkantilen Wirtschaft wie der Salinen und Metallhütten hatten die staatlichen Organe im Zuge der europaweit grassierenden „Holznot“ damit begonnen, den Wald, der über Jahrhunderte den Brennholzbedarf von armen Gelegenheitsnutzern gedeckt hatte und dem (genossenschaftlich organisierten) bäuerlichen Waldgewerbe zugänglich gewesen war, vollständig in einen staatlich kontrollierten Ort exklusiver Holzproduktion zu verwandeln. Das Beispiel macht deutlich, wie eng kapitalistische Dynamik, ökologische Krisen, soziale Konflikte und historische Projekte der Herrschaftssicherung miteinander verwoben sind (vgl. Sieferle 1982; Radkau 1983, 2008, S. 73ff.).

Wenn indes die Belastungsgrenzen regionaler ökologischer Systeme erreicht oder überschritten waren, wurden Krisen der lokalen ökologischen Entwicklung in der Ge- schichte des Kapitalismus bislang immer durch geografische Expansion und eine schnelle Inwertsetzung von neuem Land und neuer menschlicher Arbeitskraft, sowohl innerhalb wie außerhalb Europas, gelöst. Daher wanderte die "frontier" (Grenze) der Zuckerproduktion im 16. und 17. Jahrhundert von Madeira nach Brasilien und später auf die Karibischen Inseln; die „frontier“ des Silberbergbaus verlagerte sich aus dem erschöpften Erzgebirge nach Peru. Im 16. und 17. Jahrhundert verhalf die Eroberung der „frontiers" für Nordseefisch, norwegisches Holz, brasilianischen Zucker, peruanisches Silber und polnisches Getreide den Amsterdamer Kaufleuten dazu, zum Organisator und Kapitalgeber des transatlantischen und innereuropäischen Schiffsverkehrs zu werden (Moore 2010b), im 19. bzw. 20. Jahrhundert bildete die Eroberung der „frontiers“ für Baumwolle und Erdöl eine wichtige Grundlage für den Aufstieg zunächst Englands, sodann der USA zur Weltmacht.

\section{Das Ende der billigen Produktionsinputs}

Das Öl war und ist auch heute noch ein ganz besonderer Stoff, geeignet für so viele industrielle und landwirtschaftliche Verwendungsmöglichkeiten wie kein anderer mineralischer Rohstoff. In den 1930er Jahren, als die Förderung der damals noch dicht unter der Erdoberfläche komprimierten Ölreserven erstmals über 200 Mio. Tonnen pro Jahr angestiegen war, lag der Energieertrag des einheimischen Öls in den USA noch bei $100: 1$, d.h. es wurde nur eine Einheit Energie benötigt, um hundert Energieeinheiten Öl zu fördern. Bei dem heute in Nordamerika geförderten „unkonventionellen“ Öl aus Teersanden oder dem Schiefergas, das mithilfe der umstrittenen Technologie des "Fracking“ gefördert wird, liegt dieser Wert aber lediglich bei $11: 1$ bis 18: 1 (im Falle des Schiefergases) bzw. bei 1: 1 (im Falle der Teersande, vgl. Mórrigan 2010). Nur Energieträger mit einem hohen Energieertrag ermöglichen aber hohe Zuwachsraten der Produktivität der Arbeit und daher auch hohe Wachstumsraten. Nach dem Erreichen von „Peak Oil“ und „Peak Gas“, also dann, wenn das Produktionsplateau bei konventionellem Öl und Gas überschritten wird, ist die Erschöpfung dieser Energieträger unwiderruflich. Wann sie eintritt, hängt davon ab, wie schnell und effektiv die Ausbeutung erfolgt. Gleichzeitig steigt der Druck, neue Ressourcen an „unkonventionellem“ 'Öl und Gas zu erschließen. Ob diese aber tatsächlich auch gefördert werden, hängt von der Verfügbarkeit entsprechender Technologien ab, vom kostengünstigen Zugang zu Energie und (im Falle des Fracking) auch von Zugriffsmöglichkeiten auf sehr große Mengen von Wasser.

Vor allem aber setzt dies einen hohen und in näherer Zukunft weiter steigenden Ölpreis voraus. Letztlich bestimmt dieser darüber, ob es wirtschaftlich sinnvoll ist, eine 
durch Bohrungen bestätigte (unkonventionelle) Ressource in eine „nachgewiesene Reserve“ $z$ u verwandeln und bei ausreichender Finanzierung mit der Förderung zu beginnen. Die Befolgung einzelwirtschaftlicher Rationalität kann auch dazu führen, dass die Quellen von „unkonventionellem“ Öl und Gas mit Hilfe der neuesten Technologie (und selbstverständlich ohne Rücksicht auf irgendwelche Umweltbelange) besonders schnell geleert und die Energieträger zu niedrigen Preisen verkauft werden - so wie dies gegenwärtig im Zeichen des (auf wenige Jahre projektierten) „Fracking-Gas-Hypes“ in den USA geschieht (Rogers 2013). Wenn dann, wie im Falle des US-amerikanischen Schiefergases und -öls, die günstig ausbeutbaren Vorkommen, die auf gerade einmal 5 bis $10 \%$ aller Ressourcen geschätzt werden (Hughes 2013), schnell zu Ende gehen, kann nur ein hoher und steigender Preis dafür sorgen, mit der extrem teuren Exploration und im günstigsten Fall nachfolgender Förderung fortzufahren.

Der unaufhaltsame Anstieg der Energiekosten wird auf diesem Wege aber nicht gebremst. Daher geht die Internationale Energie Agentur (IEA 2013) auch davon aus, dass der Preis für ein Barrel importierten Rohöls mittelfristig weiter steigen wird. Hohe Energiepreise aber gehen prinzipiell nicht mit hohem ökonomischen Wachstum zusammen; sie befördern eher wirtschaftliche Rezessionen. Auch die erneuerbaren Energieträger Sonne, Wind und Geothermik, die gegenwärtig noch nicht einmal $5 \%$ des globalen Nettoenergieverbrauchs ausmachen, können in absehbarer Zeit keine billigen Energielieferanten sein, zumal dann nicht, wenn die mächtige „fossile Lobby“ ihren Ausbau bremst, um die in „braunen“ Industriezweigen getätigten Anlagen profitabel zu halten. Doch nur billige Produktionsinputs können die organische Zusammensetzung des Kapitals günstig beeinflussen und den von technologischen Innovationen und Lohnsteigerungen ausgehenden Druck auf die Profite eindämmen. Daher waren die Profite in der Geschichte des Kapitalismus auch immer dann hoch und ausreichend für neue Investitionen, wenn natürlicher Reichtum im Überfluss zur Verfügung stand: große Wälder und leicht zugängliche Kohleflöze in Europa, ergiebige Silber- und Kupferadern in Lateinamerika, riesige Ölfelder dicht unter der Erdkruste in den USA, am Kaspischen Meer oder auf der arabischen Halbinsel, dicke Schichten von Naturdünger an der Pazifikküste, weite fruchtbare Flächen für den Getreideanbau im Mittleren Westen der USA.

Daraus folgt nun aber keineswegs, dass für die Stabilität des Kapitalismus als sozioökonomisches System die absolute, physische Erschöpfung von biophysischen Systemen ausschlaggebend wäre; die Missachtung von biophysischen Grenzen gehört ja zu den Wesensmerkmalen des Kapitals. Kritisch aber wird es, wenn Böden, Wälder, Ozeane und Minen einerseits, menschliche Arbeitskraft andererseits nur unter Einsatz von mehr Energie und mehr Technologie und daher von mehr Kapital ausgebeutet werden können. Denn dies begrenzt das Wachstum, die Vermehrung des Kapitals um seiner selbst willen. Und dann lassen sich die monopolistischen Privilegien bestimmter Standorte nicht mehr auf- rechterhalten. Genau dies geschieht aber mit der fortschreitenden Integration immer neuer „Boom“-Regionen in den Weltmarkt: Wenn es keine große „frontier“ mehr gibt, sondern nur Territorien, die bereits dem vom Weltmarkt ausgehenden Wettbewerb unterworfen sind, verlieren die vormaligen Quellen leicht realisierbaren Profits ihren einstigen Vorteil - eben den, nicht-kommodifiziertes, also dem Wertgesetz (noch) nicht unterworfenes „Neuland“ zu sein - und es versiegt die Quelle leicht realisierbaren Profits.

Auch im modernen Kapitalismus der Hightech-Ära bleibt die Dynamik der Akkumulation abhängig von den „Geschenken“ der bio-physikalischen Systeme - von der kostengünstigen Verfügbarkeit von Sand, ohne den keine Stahl-Beton-Konstruktion möglich ist; von Phosphat, ohne das die industrielle Landwirtschaft nicht auskommt; von Seltenen Erden und anderen nur in geringer Konzentration in der Erdkruste vorkommenden Metallen, ohne die kein Windrad, kein Handy und auch keine Drohne funktionieren würde. Wenn zukünftig mit Hilfe der „blauen Biotechnologie" auch der Grund der Meere zu einer Industrielandschaft umgestaltet werden sollte - und Rohstoffe aus der Tiefsee genutzt würden, um Öl, Ersatzstoffe für die chemische und die pharmazeutische Industrie, Nahrungsergänzungsstoffe, Biochemikalien oder Biogas herzustellen -, so wäre doch der „energy return on energy invested“ und damit auch der „capital return on capital invested“ absehbar so gering, dass ein gewaltiger neuer Wachstumsschub davon nicht angestoßen werden könnte.

\section{Kapitalismus an den Wendepunkten der biophysischen Systeme}

Auf der Ressourcenseite muss nicht allein mit ökonomischer Knappheit gerechnet werden, die sich in zunehmender Preisvolatilität und unkalkulierbaren Marktentwicklungen ausdrückt. Vielmehr sind physische Grenzen absehbar, die sich aus der schwindenden Verfügbarkeit von technisch leicht erschließbaren und daher kostengünstigen Ressourcen ergeben. Unter diesen Bedingungen aber kann die im Kapitalismus als dynamisches Prinzip gepriesene Knappheit schnell in elementaren physischen Mangel an essenziellen Gütern umschlagen - in einen Mangel an Energie, Nahrungsmitteln oder Wasser für die unter Bedingungen steigender Rohstoffpreise nicht (mehr) zahlungsfähigen Marktteilnehmer. Dieses Szenario gewinnt an Schärfe, wenn geopolitische Dimensionen der Knappheit hinzukommen, ausgelöst etwa durch Exportrestriktionen unter den Bedingungen eines wachsenden „Rohstoffnationalismus“ oder als Folgen der zunehmend schwieriger werdenden Kontrolle sogenannter "Konfliktregionen“.

Daher ist bereits von der „,input“-Seite kapitalistischer Produktion und Konsumption her zu bezweifeln, dass dieses System in seiner modernen Form als ein globalisiertes 
Produktionssystem auch zukünftig zu einer ins Unendliche strebenden Bewegung fähig ist. Denn der Kapitalismus ist auf die stets wachsende Aneignung einerseits von „Gratisleistungen der Natur", andererseits des abstrakten Reichtums in der Form des Geldes angewiesen. Heute zeichnen sich aber nicht allein auf der Ressourcenseite absolute Grenzen der Natur ab, die nur im Konflikt und unter Einsatz von Gewaltmitteln zum Schutz partikularer Interessen überschritten werden können; die Mechanismen des Marktes werden nicht hinreichen, um für einige Wenige den exkludierenden Zugang zu unentbehrlichen Stoffen und Energieträgern zu sichern. Gravierender und unvermeidlicher als die sich anbahnenden Konflikte um Rohstoffe aller Art sind die Grenzen, die sich auf der Schadstoffseite der kapitalistischen „Weltvernichtungsmaschine“, bei der Überfüllung der natürlichen „Senken“ (v. a. für klimaschädliche Gase), abzeichnen.

Der biophysische Blick auf die Ökonomie lehrt zum einen, dass wir nicht Etwas aus Nichts machen können - und daher mit den endlichen Ressourcen zurechtkommen müssen, die in der Erdkruste stecken; und dass wir die erneuerbaren Ressourcen Land, Wasser und komplexe Biosysteme nur soweit vernutzen können, wie diese sich selbst zu reproduzieren vermögen. Zum anderen lehrt ein solcher Blick, dass unsere Form des Wirtschaftens, der Mobilität, des Wohnens, Konsumierens und Kommunizierens jede Menge „Abfallprodukte“ erzeugt, die nicht einfach verschwinden; sie lagern sich als klimaschädliche Gase in der Erdatmosphäre oder in den Weltmeeren ab und drohen die planetarischen Grenzen unserer biologischen und physikalischen Systeme zu überschreiten.

Wenn indes „Kippschalter“ dieser Systeme aktiviert werden, kann der Kollaps komplexer Ökosysteme sehr rasch eintreten - und er wäre irreversibel. Das Abschmelzen des Grönlandeises und des westarktischen Eisschildes ist einer dieser „tipping points“, denn in ihrer Folge wird der Meeresspiegel langfristig um mehrere Meter ansteigen; ebenso wie das Umkippen der thermohalinen Zirkulation im Nordatlantik die Durchschnittstemperatur in Europa deutlich senken und zum Austrocknen des Amazonasregenwaldes führen würde. Das wird der Planet verkraften, doch sind die Folgen für die menschliche Spezies unabsehbar: „Eine Aktivierung eines jeden dieser Kippschalter könnte das Habitat, in dem die Menschheit seit dem Holozän lebt, schwer schädigen oder gar zerstören“" (Edenhofer 2012, S. 272). Wie zwei jüngst veröffentlichte US-amerikanische Studien dokumentieren, scheint der „point of no return" für das Schmelzen des mächtigen westantarktischen Eisschildes inzwischen überschritten zu sein (vgl. The Observer vom 17. Mai 2014 und New York Times vom 30. Mai 2014), und auch der Regenwald des Amazonas-Gebiets nähert sich - trotz in den letzten Jahren reduzierter Abholzungsraten - in erschreckendem Tempo jenem Punkt, der das System zum Kippen bringen und eine großflächige „Savannisierung“ einleiten könnte.

An solchen erdgeschichtlichen Wendepunkten wird sich das kapitalistische „business as usual“ nicht aufrechterhalten lassen. Allein das mittlerweile nahezu unabwendbare Über- schreiten einer Erderwärmung von mehr als zwei Grad Celsius gegenüber dem vorindustriellen Niveau wird eine Kaskade katastrophischer Veränderungen auslösen: extreme Hitze, die über einigen Landflächen zu durchschnittlichen Temperaturerhöhungen von $4^{\circ}$ bis $10^{\circ} \mathrm{C}$ führen wird; eine substanzielle Verschärfung der Wasserknappheit in vielen Weltregionen (darunter die gesamte Mittelmeerregion) und häufige Überflutungen in anderen Regionen, mit dramatischen Folgen für die Nahrungsmittelproduktion; einen Anstieg des Meeresspiegels um einen halben bis einen Meter, von dem vor allem hunderte Millionen Menschen in den dicht besiedelten Städten des globalen Südens betroffen sein werden; eine zunehmende Wucht von Wirbelstürmen, die etwa den nordamerikanischen Kontinent treffen wird. Auch die Folgen des Überschreitens von planetarischen Grenzen bei den biologischen Ressourcen und der Übersäuerung der Weltmeere - beides scheint kaum noch vermeidbar - wird mit ähnlich einschneidenden Folgen verbunden sein.

\section{Schlussfolgerungen}

Ökonomisches Wachstum bedeutet auf jeweils gegebenem technischem Niveau stets wachsenden Ressourcenverbrauch und wachsende Schadstoffemissionen - auch wenn seit Jahren das Lied von der „Entkopplung“ der Ressourcen- und der Schadstoffströme vom ökonomischen Wachstum gesungen wird (vgl. kritisch Mahnkopf 2012). Unter den Bedingungen kapitalistischer Wettbewerbsdynamik und Profitmaximierung kann es gar nicht anders sein, als dass Einsparungen bei Energie- und Rohstoffverbrauch, die sich durch technische Fortschritte erzielen lassen, umgehend in vermehrte Produktion umgesetzt werden, selbst wenn diese zu gewaltigen Überkapazitäten von allem und jedem führt - von Autos, Informationselektronik und nahezu sämtlichen langlebigen Konsumgütern. Daher verwundert es nicht, dass trotz erheblicher Effizienzsteigerungen, die während der letzten Jahrzehnte bei der Verwendung von Energie und Stoffen erzielt worden sind, sowohl Ressourcenverbrauch wie Emissionen weiter ansteigen. Eine Dekarbonisierung der Weltwirtschaft um annähernd $85 \%$ in den Industrie- und ca. $50 \%$ in den Entwicklungsländern - wie in dem Vierten Bericht des Intergouvernemental Panel on Climate Change (IPCC) aus dem Jahr 2007 für nötig erachtet, um die globale Erwärmung bei $2^{\circ}$ bis $2,4^{\circ} \mathrm{C}$ gegenüber dem vorindustriellen Niveau stabilisieren zu können, - ist mit den Mitteln des technischen Fortschritts, mit freiwilligem Konsumverzicht und einigen Veränderungen der politischen Regulation schlichtweg nicht machbar.

Selbst wenn es auf der Rohstoffseite des Kapitalismus weder ökonomische noch physische oder geopolitische Knappheit gäbe, die über kurz oder lang zu elementarem Mangel für wachsende Teile der Weltbevölkerung füh- 
ren müsste, auf welchen schwerlich mit bloßer Schicksalsergebenheit reagiert werden dürfte: Die absoluten Grenzen auf der Schadstoffseite des Systems lassen sich noch weniger ignorieren. Denn die Deponien für den Abfall der nach Prinzipien kapitalistischer Steigerungslogik organisierten Prozesse der Stoff- und Energietransformation sind schlichtweg voll. Werden die bereits heute eingepreisten fossilen Energiereserven tatsächlich gefördert und verbrannt, ist ein Anstieg der Erdmitteltemperatur um $4^{\circ}$ bis $5^{\circ} \mathrm{C}$ unvermeidlich.

Daher hängt letztlich alles davon ab, ob es innerhalb der kurzen Zeitspanne von zwei bis drei Jahrzehnten gelingen wird, unsere sozialen und ökonomischen Systeme so radikal umzubauen, dass der Ressourcenverbrauch und die Schadstoffproduktion, die mit den spezifisch kapitalistischen Formen des Wirtschaftens, der Mobilität, des Wohnens, des Konsumierens und der Kommunikation verbunden sind, innerhalb der planetarischen Grenzen von biologischen und physikalischen Systemen bleiben. Gelingt dies nicht, dann erledigt sich die Frage nach der Zukunft des Kapitalismus als eines weltökologischen Systems auf eine erdgeschichtlich radikale Weise. Denn es werden unter diesen Bedingungen nicht nur der Welthandel und die Zerstreuung der Produktion über den ganzen Globus ein Ende finden. Es würde sich ein derart großes Katastrophenpotenzial aufbauen, dass große Bereiche menschlicher Zivilisation schlichtweg kollabieren könnten. Gemessen an dieser Perspektive erscheint eine sozialgeschichtlich radikale Wende als ein zwar schwieriges, gleichwohl aber überlebensnotwendiges Projekt. Eine Wende, die dafür sorgt, dass Öl, Gas und Kohle im Boden bleiben und Desinvestment bei den fossilen Energieträgern wie auch bei vielen anderen Rohstoffen politisch erzwungen wird - und folglich ökonomisches Wachstum sich wieder auf jenes homöostatische Gleichgewicht einpendelt, das für Jahrtausende menschlicher Zivilisation auf dem Planeten charakteristisch war.

\section{LITERATUR}

Acemoglu, D./Robinson, J. A. (2012): Why nations fail: The origin of power and prosperity, New York

Altvater, E. (2005): Das Ende des Kapitalismus, wie wir ihn kennen, Münster Altvater, E. (2014): Anthropozän. Steigerungsformen einer zerstörerischen Wirtschaftsweise, in: Emanzipation 3 (1), S. 71-88

Bardi, U. (2013): Der geplünderte Planet. Die Zukunft des Menschen im Zeitalter schwindender Ressourcen, München

Crutzen, P. (2002): Geology of mankind. The anthropocene, in: Nature (415), S. 3 Davis, M. (2007): Planet der Slums, Berlin

Dreze, J./Sen, A. (2013): An uncertain glory: India and its contradictions, Princeton Edenhofer, O. (2012): Globale Energiewende -Wege zu einer nachhaltigen Energieversorgung, in: Debiel, T./Hippler J./Roth, M./Ulbert, C. (Hrsg.): Global Trends 2013. Frieden - Entwicklung - Umwelt, Frankfurt a. M., S. 265-284

Hughes, D. J. (2013): Drill, baby, drill. Can unconventional fuels usher in a new era of energy abundance?, Post Carbon Institute, http://www.postcarbon.org/ reports/DBD-report-FINAL.pdf

International Energy Agency (IEA) (2013): World energy report 2013, Paris Konicz, T. (2012): Automatisches Subjekt. Dem Kapitalverhältnis wohnt ein Wachstums- und Verwertungszwang inne, in: Junge Welt vom 4. April

Maddison, A. (2001): The world economy: A millennial perspective, Paris Mahnkopf, B. (2012): Kapitalismuskritik als Wachstumskritik, in: Dörre, K./Sauer, D.Wittke, V. (Hrsg.): Kapitalismustheorie und Arbeit. Neue Ansätze soziologischer Kritik, Frankfurt a. M., S. 389-409

Mahnkopf, B. (2013): Peak everything - peak capitalism? Folgen der sozialökologischen Krise für die Dynamik des historischen Kapitalismus: DFG-Kollegforscherlnnengruppe Postwachstumsgesellschaften, Working Paper 02/2013, Jena, http://www.kolleg-postwachstum.de/sozwgmedia/dokumente/ WorkingPaper/wp2_2013.pdf
Marx, K. (1970): Das Kapital. Kritik der politischen Ökonomie, MEW Bd. 23, Berlin Moore, J.W. (2010a): "Amsterdam is standing on Norway", part I: The alchemy of capital, empire and nature in the diaspora of silver, 1545-1648, in: Journal of Agrarian Change 10 (1), S. 33-68

Moore, J. W. (2010b): "Amsterdam is standing on Norway", part II:The global North Atlantic in the ecological revolution of the long seventeenth century, in: Journal of Agrarian Change 10 (2), S. 188-227

Moore, J. W. (2012): Crisis: Ecological or world-ecological?, in: Wiedemann, C./ Zehle, S. (Hrsg.): Depletion design: A glossary of network ecologies, S. 73-78 Mórrigan, T. (2010): Peak energy, climate change and the collapse of global capitalism, University of California, Santa Barbara, http://energyskeptic. com/2012/tariel-morrigan-peak-energy-climate-change-and-the-collapse-ofglobal-civilization/

Pikkety, T. (2014): Capital in the twenty-first century, Cambridge/Mass.

Radkau, J. (1983): Holzverknappung und Krisenbewußtsein im 18. Jahrhundert, in: Geschichte und Gesellschaft 9 (4), S. 513-543

Radkau, J. (2008): Technik in Deutschland. Vom 18. Jahrhundert bis heute, Frankfurt a. M.

Rockström, J. et al. (2009): Planetary boundaries: Exploring the safe operating space for humanity, in: Ecology and Society 14 (2), http://www.ecologyandsociety. org/vol14/iss2/art32/

Rogers, D. (2013): Shale and Wall Street: Was the decline in natural gas prices orchestrated? Energy policy forum, http://energypolicyforum.org/portfolio/ was-the-decline-in-natural-gas-prices-orchestrated/

Sieferle, R.-P. (1982): Der unterirdische Wald. Energiekrise und industrielle Revolution, München

Sombart, W. (1987): Der moderne Kapitalismus, Endgültige Auflage, PaperbackReprint, 3 Bde., München

United Nation Development Program (UNDP) (2014): Humanity divided: Confronting inequality in developing countries, New York

\section{AUTORIN}

BIRGIT MAHNKOPF, Dr. habil., ist Professorin für Europäische Gesellschaftspolitik an der Hochschule für Wirtschaft und Recht (HWR) und Mitglied des Institute for International Political Economy Berlin (IPE Berlin). Arbeitsschwerpunkte: Ökonomische, soziale und politische Dimensionen der Globalisierung; Politische Ökonomie der europäischen Integration; Arbeitssoziologie und Industrielle Beziehungen; Bildungs- und Gesellschaftspolitik.

mahnkopf@hwr-berlin.de 